\title{
LE CHRISTIANISME À VENIR
}

\author{
Christianity to come
}

Paul Gilbert SJ *

Résumé: Pour penser l'avenir, il convient d'examiner le présent, les mouvements qui s'y dessinent et qui laissent prévoir la formation du futur. En présentant quelques auteurs francophones qui ont tenté de discerner les enjeux vécus par l'Église depuis le concile Vatican II, l'article met en évidence l'importance de la rencontre de $1^{\prime \prime}$ "autre" et de son écoute. Beaucoup d'auteurs critiquent en effet l'Église officielle qui écoutait peu et imposait beaucoup. La crise que connaît le christianisme contemporain peut être lue de cette manière, une crise des relations humaines en société, en Église. C'est ce que firent des auteurs comme François Roustang, Georges Morel, Maurice Bellet, Stanislas Breton, et quelques autres.

Paroles clés: Bellet. Christianisme. Morel. Roustang. Vatican II.

Abstract: Thinking about the future requires examining the present and the unfolding movements that foretell the shape of the future. By presenting a few French-speaking authors, in their attempt to discern the issues experienced by the Church since the Second Vatican Council, the article highlights the importance of meeting and listening to the "other". Many authors criticize the official Church that listened little and demanded much. The contemporary crisis of Christianity can be understood as a crisis of human relations in society and in the Church. This is how authors like François Roustang, Georges Morel, Maurice Bellet, Stanislas Breton, and a few others see it.

Keywords: Bellet. Christianism. Morel. Roustang. Vatican II.

* Professor titular de filosofia da Pontifícia Universidade Gregoriana, Roma. Artigo recebido em 15/11/2020 e aprovado para publicação em 20/11/2020. 


\section{Introduction}

$\mathrm{L}$

e titre proposé pour cet article imite le titre d'un des derniers ouvrages de Stanislas Breton ${ }^{1}$. Le propos est provoquant. Ne dit-on pas aujourd'hui que le christianisme est maintenant en fin de vie, qu'il a accompli sa raison d'être ? Les églises ne sont-elles pas en effet de plus en plus vides, et beaucoup de chrétiens, même de vieille souche, ne sont-ils pas en train de s'en aller, parfois dans des sectes ? Arrivés à la fin du christianisme, ne sommes-nous pas à la fin de l'histoire, ou d'une histoire qui n'a plus besoin du christianisme ? N'y a-t-il plus rien à espérer du christianisme, alors que celui-ci, comme le judaïsme, est orienté vers un avenir de toute la création promis par la Bible, le livre de ceux qui sont en « attente de Dieu $»^{2}$ ou du « retour » du Christ ?

Notre époque est en crise, moins maintenant sous la poussée des sciences modernes que d'une communication globalisée où les opinions de chacun sont émises dans l'immédiat de l'instant en revendiquant un droit égal à celui des auteurs dont le langage est réfléchi, construit, critique de soi et participé, base de quelque scepticisme envers soi-même ou de quelque humilité envers la société, conditions aussi pour penser l'avenir. Notre époque veut un immédiat quotidien. Sont particulièrement en cause les liens du corps et de la liberté, de la parole et de l'esprit, tous éléments constitutifs de toute anthropologie complète. L'immédiat a envahi nos pensées, et tout espoir se réduit à vivre nos passions élémentaires sans conséquences

Le christianisme aura-t-il encore droit de cité dans cette ambiance mentale ? Le thème du cahier Le futur de la religion est bien plus vaste que ne l'indique le titre de mon article. Je restreins ma réflexion au christianisme, en ayant en tête une distinction proposée par Joseph Moingt dans L'esprit du christianisme, d'où je tire ces lignes : " nous nous interrogeons sur l'avenir du christianisme, un avenir qui menace de lui faire bientôt défaut s'il s'identifie à celui de la religion chrétienne, de moins en moins pratiquée $»^{3}$. Ces lignes font écho à la distinction classique, que nous retrouverons souvent dans cet article, entre la foi et la religion. Par « christianisme ", on entend un acte de liberté qui s'engage pour des valeurs transcendantes reçues d'une révélation et accueillies dans la foi. On voit déjà par-là combien le « christianisme » est loin de la mentalité aujourd'hui commune et imposée par des rythmes de productions industrielles de biens qui nous enlèvent la liberté d'être soi et de disposer de soi. Le temps du christianisme est en conflit avec le temps industriel qui a confisqué aux

\footnotetext{
${ }^{1}$ BRETON, St. L'avenir du christianisme. Paris: Desclée de Brouwer, 1999.

${ }^{2}$ WEIL, S. Attente de Dieu. Paris : Fayard, 1966.

3 MOINGT, J., L'esprit du christianisme. Paris: TempsPrésent, 2018, p. 33.
} 
personnes le temps de la religion, et sa bonté, pour le remplacer par le plaisir de la consommation à portée de main.

Cette crise semble récente, mais elle est préparée de longue date. Mon exposé considérera des ouvrages en langue française publiés dès la fin de Vatican II par des auteurs, jésuites mais pas seulement, qui ont subis, parfois à leur dépends, le choc de l'abandon de la pratique religieuse de la part du peuple chrétien. Le discrédit de la religion en général et du christianisme en particulier vient aussi, outre aux changements des rythmes de vie et de mentalité, des interprétations d'auteurs du 19e siècle que Paul Ricœur a appelé les «maitres du soupçon »" Nous introduirons notre réflexion en rappelant quelques données élémentaires venues de ces soupçons, et nous proposerons en alternative une réflexion de Simon Decloux qui reprend la proposition de Ricœur en mettant l'accent sur la signification des vœux de religion. Nous aurons ainsi disposé une sorte de sic et non introductif au débat qu'affrontera notre article. Après quoi commencera notre lecture d'auteurs (le sociologue Marcel Gauchet, et des commentateurs de mystiques comme François Roustang et Georges Morel) pour qui le christianisme comme religion ou théorie a manifestement terminé sa course. Nous verrons ensuite que cet avis doit être nuancé, car le christianisme n'est pas que formulations théoriques. Il requiert une attention à une intériorité originaire, à une demande de sens qui, selon Luc Ferry, n'est pas sans une requête du sacré, sans aussi la reconnaissance d'une bipolarité de désir de justice et de fondement abyssal (Jacques Derrida en montre l'essentialité dans les religions). Au christianisme, selon Maurice Bellet, appartient enfin un déploiement phénoménologique de l'écoute d'autrui. Nous terminerons cet exposé en revenant à Stanislas Breton.

\section{Un débat}

Durant les XIXe et XXe siècles, beaucoup plus que lors de l'Auklärung ou des encyclopédistes comme Diderot et D'Alembert, ou Voltaire, la critique de la religion s'est développée d'une façon scientifique, au sens où elle est analysée selon les canons scientifiques de l'Instauratio Magna de Francis Bacon, vieux mais toujours d'actualité, du début du XVIIe siècle. La science travaille en établissant trois tables entre deux événements observables, une qui note la concordance entre des modifications de ces événements (si A se modifie en A1, B se modifie également en B1), une autre la proportion entre les modifications d'un événement et de l'autre (A1 vaut 3 points, mais B1 en vaut le double, 6), et la troisième qui constate une absence de relation entre ces événements (B reste $B$

\footnotetext{
${ }^{4}$ Voir RICCEUR, P. De l'interprétation : Essai sur Freud. Paris: Seuil, 1965, p. 42.
} 
même si A devient A1). Les maîtres du soupçon adoptent ces «tables ». Marx note par exemple une évolution parallèle entre un état social et la confiance qu'on donne à des idées religieuses, Freud entre les représentations de soi et la reconnaissance de soi, et Nietzsche entre la volonté de puissance et la qualité de l'amour de soi. Toute religion, mais surtout le christianisme, ne serait qu'» un reflet (Marx), une illusion (Freud) ou un masque (Nietzsche), sous lesquels se cache une réalité essentielle qu'il s'agit précisément de mettre à nu $»^{5}$.

Le cas de Marx est sans doute le plus clair. Le libéralisme et la propriété privée créent des classes sociales dont l'antagonisme peut éclater au grand jour si une personnalité extraordinaire en fait prendre conscience à ceux qui sont utilisés par des propriétaires sans règles, et anime leur action de protestation. Il en va de même en psychanalyse, où des diagnostiques peuvent porter à des résultats de guérison. Les maîtres du soupçon révèlent ainsi des dynamiques causales qui requièrent en correspondance des actions précises. Il y a ainsi à l'évidence des relations entre les situations humaines et les représentations religieuses. Les sciences humaines éclairent ces corrélations entre des événements humains et les rites religieux, quitte à faire correspondre les secondes au premiers, et à soutenir ensuite que ceux-ci sont les « causes » des secondes. Sur ces questions, y compris celle du cercle vicieux que nous venons de dénoncer, les sciences humaines continuent leurs recherches empiriques avec la même vieille méthode des corrélations et de leurs interprétations par la causalité, jusqu'à soutenir l'idée générale que la pauvreté mentale est la cause de l'acceptation des idées et de la dévotion religieuse.

En faisant reconnaître les exagérations unilatérales de ces herméneutiques réductrices, Ricœur souhaite orienter ses lecteurs vers des herméneutiques instauratrices, à partir desquelles une espérance devient possible 6 . Un essai de Simon Decloux, qui fut assistant du Père Général des jésuites Pedro Arrupe, va dans cette direction. Ricœur distinguait les trois maîtres du soupçon en attribuant à chacun d'eux un des domaines les plus essentiels de l'existence humaine : l'avoir (Marx), le pouvoir (Freud) et le valoir (Nietzsche) ${ }^{7}$. Or à ces trois domaines (qui caractérisent aussi les trois tentations de Jésus au désert dans la version lucanienne - Lc 4,1-12) correspondent les trois vœux de religion, la pauvreté (le rapport au monde), la chasteté

\footnotetext{
${ }^{5}$ DECLOUX, S. “Vie religieuse et « Maîtres du soupçon »". In: Vies religieuses 50, 1978, p. 195225. Nous indiquerons directement dans notre texte la pagination du texte cité. Dans le cas de Decloux, nous citons l'édition informatique de cet article (voir la page web de la revue). ${ }^{6}$ Voir RICCEUR, P. Le conflit des interprétations : Essais d'herméneutique. Paris : Seuil, 1969, p. 393-415("La liberté selon l'espérance"). « De cette liberté [religieuse], il y a herméneutique, dans la mesure où le phénomène religieux lui-même n'existe que dans le procès historique de l'interprétation et de la réinterprétation de la parole qui l'engendre » (p. 393).

${ }^{7}$ Voir P. RICEEUR, « Le $\theta u \mu o \varsigma$ : avoir, pouvoir, valoir ». In : RICCEUR, P. L'homme faillible. Paris : Aubier-Montaigne, 1960, 122-142.
} 
(le rapport à l'autre), l'obéissance (le rapport à Dieu). Il s'agit à chaque fois de mettre en valeur une attitude humaine, une manière d'être " en rapport à ». Voyons ce qu'en dit Decloux ${ }^{8}$.

D'abord la pauvreté et le rapport au monde.

Nous voyons $[\ldots]$, dans notre vœu de pauvreté, l'importance de l'attitude spirituelle de gratuité. Il y a dès lors, dans cet univers que j'ai essayé d'évoquer en partant de Marx, l'exigence d'établir une véritable fraternité dès à présent, pas demain seulement, non la fraternité qui suivra la révolution, mais celle du partage dès aujourd'hui, avec tous, autant que possible (6-7).

Ensuite la chasteté et les relations affectives, dont la prière révèle le fond donateur.

Car s'il y a un lieu où l'affectivité de l'homme peut être touchée autrement que par ses semblables, peut être atteinte directement par Dieu, c'est précisément celui de la prière. Elle n'est pas une manière de se trouver avec soi-même pour se parler à soi-même, elle est un lieu où l'on essaie de se vider de soi pour accueillir une autre présence, une autre parole et ainsi se livrer affectivement à celui qui nous appelle et, par le fait même, peut nous combler au-delà de tout désir (13).

Enfin l'obéissance et l'être envers Dieu.

Si vivre avec une âme d'enfant consiste à rester infantile, nous ne sommes pas encore à l'âge de la vraie liberté. Encore s'agit-il de vivre l'obéissance religieuse à l'opposé de tout infantilisme, de tout étouffement, de tout écrasement de la liberté. Pour nous, il n'y a rien qui soit plus libérateur que de vivre l'obéissance, rien qui doive susciter davantage notre capacité d'initiative, de recherche, de réalisation que de nous en remettre précisément à Dieu et à ceux qui nous parlent en son nom pour guider notre vie (20).

En résumé : "Le débat est donc, en fin de compte, un débat entre des anthropologies. Il s'agit de faire apparaître une anthropologie chrétienne où l'homme découvre quelle est sa vraie dimension, sa vraie profondeur » (21).

La différence entre les maîtres du soupçon et Decloux tient en ceci : avec Decloux, la vie religieuse établit des rapports instaurateurs ou d'alliance et non conflictuels ou destructeur entre une personne et d'autres personnes, le monde et Dieu ; importe ici l'acte personnel de liberté qui entre en relation. Pour les "maîtres du soupçon ", la relation doit être défaite, ce qui laisse la personne dans la solitude d'une liberté formelle qui doit combattre pour se maintenir. Dans la typologie que nous mettrons en jeu, on dira que le soupçon fonctionne au plan l'herméneutique réductrice de la relation, et la liberté au plan instaurateur de l'acte de foi.

${ }^{8}$ S. DECLOUX, "Vie religieuse et "Maîtres du soupçon" ». 


\section{Un christianisme en fin de course}

Après la disposition des pôles en débat, le sic et non de notre argument, considérons quelques auteurs pour qui le christianisme a terminé de jouer son rôle et peut être ignoré pour construire nos vies.

\subsection{Marcel Gauchet}

Le premier auteur est Marcel Gauchet. Les maîtres du soupçon et leur herméneutique réductrice avaient comme projet de défaire ou de démolir le christianisme, sans envisager la possibilité d'autres approches que la leur. Gauchet vise plutôt à le déconstruire, ou plutôt à déconstruire les représentations qu'il s'est données au fil des siècles. Le christianisme est considéré ici comme un phénomène historique, et non plus de structures en quelque sorte abstraites du temps. Il y aura toujours des luttes de classes puisque les hommes doivent travailler la terre pour y vivre selon leurs capacités propres et diverses les unes des autres ; il y aura toujours des rapports difficiles du petit garçon avec son père ; il y aura toujours des rapports de puissance qui se heurteront à un mur. Plutôt donc que de démolir la religion et de ne la remplacer par rien, ne pourrait-on pas inventer une herméneutique plus positive, qui ouvre au futur?

Par "déconstruction », nous entendons ce que Matin Heidegger proposait au $\S 6$ d'Être et temps : " La tâche de [déconstruction] de l'histoire de l'ontologie ${ }^{9}$. Les époques récentes portent à la visibilité les richesses cachées du passé, en exhumant sans doute des structures qui y étaient originairement imprévisibles, mais qui apparaissent progressivement, siècle après siècle. Ne pourrait-on pas en effet penser que l'histoire libère le passé de ses richesses possibles en manifestant ses intentions réelles mais inchoatives, comme une fleur qui a besoin de temps pour que sa semence puisse aboutir en elle? Dans Le désenchantement du monde, Gauchet suit cette ligne interprétative. La thèse est bien connue : le christianisme a "été la religion de la sortie de la religion $»^{10}$. Selon l'auteur en effet, avec le christianisme et sa thèse sur l'incarnation, l'histoire des religions va de l'hétéronomie sacrée à l'autonomie de l'État. À l'origine, les religions naissent d'un sentiment de dépendance d'une puissance transcendante. La vie humaine y est réglée de manière hétéronome, en dépendance de

\footnotetext{
${ }_{9}$ Voir M. HEIDEGGER, Être et temps, trad. hors commerce de Martineau, Authentica, Paris, 1985, p. 38. Le mot allemand est effectivement « Destruktion ». L'exposé insiste cependant sur l'intention positive et non négative de cette expression. Il suit en effet un ordre évocateur : on part des philosophes récents, de Kant en fait, et on régresse vers Aristote en passant par Descartes.

${ }^{10}$ GAUCHET, M. Le désenchantement $d u$ monde : Une histoire politique de la religion. Paris : Gallimard, 1985, II.
} 
la nature, de forces mystérieuses, des dieux. Puis viennent la Modernité et de la naissance du capitalisme. En recherchant ce qui en constituerait la spécificité, Max Weber note un changement profond des paradigmes culturels qu'il interprète comme un désenchantement du monde ${ }^{11}$. Gauchet suit cette l'impulsion :

La désertion des enchanteurs, la disparition du peuple des influences et des ombres sont le signe de surface d'une révolution autrement plus profonde dans les rapports entre ciel et terre, révolution au travers de laquelle il y va décisivement de la reconstruction du séjour des hommes à part de la dépendance divine (II).

Entre le passé de la naissance des religions et le présent moderne, il y a donc une «scission sans appel» (III) ${ }^{12}$.

Une religion, selon Gauchet, est en effet essentiellement hétéronome. Le " choix de se posséder en consentant à sa dépossession, en se détournant du dessein de dominer la nature et de légiférer pour son propre compte au profit d'un autre dessein, celui-là de s'assurer d'une identité de part en part définie et maîtrisée, c'est la religion dans son essence » (VIII). Le moteur de cette soumission à un pouvoir hétérogène se situe dans une mentalité archaïque " déjà pleinement civilisée » (IX) mais aussi sauvage car elle n'est pas attentive à la rigueur scientifique, à « la contrainte au résultat [qui] domestique [nos] opérations »(VIII).

Entre nous et ces sociétés antiques aux civilisations déjà complexes, " la manifestation patente de l'écart, c'est l'absence d'État, et le secret de l'identité, c'est le rôle de la religion » (IX). C'est en effet "l'émergence de l'État [qui] apparaît clairement comme l'événement majeur de l'histoire humaine » $(X)$. L'État, en s'alliant à la science, va reprendre en effet une bonne part de la fonction sociale et politique de la religion. Avec l'écriture et les observations scientifiques réussies de manière autonome, se produit comme un « séisme entraînant le basculement dans un nouvel univers tant matériel que spirituel » $(\mathrm{X})$.

Ce basculement n'est pas immédiat, mais progressif. Il faudra du temps pour que l'humanité s'y donne en acceptant d'être responsable du succès de ses propres entreprises. La création de l'État moderne entame un processus qui ira ensuite jusqu'à la révolution spirituelle qui « correspond à une redistribution pratique des termes du dispositif initial, dont le point focal est l'incarnation du séparé parmi les hommes » (XVI-XVII). L'État

\footnotetext{
${ }^{11}$ Voir WEBER, M. L'éthique protestante et l'esprit du capitalisme. trad. Grossein, Paris : Gallimard, 2003.

12 On ne pourra pas nier, dans cette thèse, l'influence de la lecture de la modernité que propose Heidegger, comme étape décisive de la progression de l'oubli de l'être. Mais aussi la thèse de BERGSON, H. Les deux sources de la morale et de la religion. Paris : Alcan, 1934, la distinction de la « religion statique » et de l'» âme ouverte ».
} 
prend alors une physionomie sacrée au sein de l'histoire des peuples, dans une culture laïque qui reconnaît l'autonomie de ses techniques scientifiques. "L'idée du divin est désormais soumise à l'effet en retour de l'action politique »(XVII).

En clôture de son ouvrage, Gauchet note cependant que la vie religieuse vécue personnellement reste significative selon ses canons anciens. Une tension demeure, ou une non identité, entre les personnes et l'État, mais d'une manière tout à fait personnelle - ce qui ne signifie pas seulement «privée ». "Entre religiosité privée et substituts à l'expérience religieuse, nous n'en aurons jamais terminé, probablement, avec le religieux » (292). Ce qui appartient à l'ordre des structures sociales n'étouffe pas toutes les exigences des personnes en société. Le religieux, en effet, « continue d'habiter les opérations de pensée, il préside à l'organisation de l'imaginaire, il gouverne les formes du problème de soi » (293).

Le livre de Gauchet est de 1985. L'auteur fait remarquer qu'il n'est ni théologien ni philosophe mais sociologue, bien que sociologue intéressé en philosophie et théologie. Son regard sur la religion n'ignore pas les changements de paradigmes qui ont marqué la philosophie et les sciences humaines autour de 1970, les affirmations structuralistes sur l'historicité de la raison et de ses expressions. La raison moderne n'est pas l'antique, ni la contemporaine. Les travaux de Michel Foucault ${ }^{13}$ ont révélé (et empêcher d'ignorer encore) cette historicité. Gauchet met en évidence des structures, mais avoue en même temps que les personnes individuelles n'en dépendent pas d'une façon nécessaire en tout. Une personne peut chercher un sens à ses actions qui ne soit pas soumis aux seules normes de l'État. Comment donc harmoniser les structures et les libertés ? Ce problème, nous l'héritons de la culture anthropologique du XIXe siècle. Nous avons déjà signalé avec Decloux que les domaines envisagés par les maîtres du soupçon pouvaient être reconnus sensés d'une autre manière qu'eux, en invitant à des engagements libres que ces maîtres n'ont pas jugé dignes d'interroger sans les réduire en forme de causalité. C'est ce manque qu'a affronté François Roustang, jésuite qui vécut personnellement une crise qui toucha beaucoup de chrétiens et de prêtres. Il s'intéressa à la psychanalyse de Jacques Lacan alors qu'il dirigeait la revue Christus, puis quitta la Compagnie et devint lui-même psychanalyste, puis spécialiste en hypnose.

\subsection{François Roustang}

Nous sommes moins d'un an après le concile Vatican II, et 20 ans avant Le désenchantement du monde de Gauchet. Roustang publie en 1966 un article

${ }^{13}$ FOUCAULT, M. Les mots et les choses. Paris : Gallimard, 1966. 
qui se fit remarquer : «Le troisième homme $»^{14}$. Vatican II a, quoi qu'on en dise, modifié ou mis au clair pas mal de non-dits qui servaient jusqu'alors à l'organisation hiérarchique de l'Église, qui était une structure politique commune dans le "siècle » où un pôle de pouvoir s'impose constitutivement aux obéissants. Les pères conciliaires entreprennent en effet une réflexion sur les évêques en lien avec une théologie du peuple de Dieu que les travaux préparatoires à leur réunion n'avaient pas prévue. Dans la constitution dogmatique Lumen Gentium, la réflexion sur l'épiscopat, et le sacerdoce en général, suit en effet et ne précède pas l'exposé sur l'essence de l'Église. La hiérarchie fait partie du peuple et n'est plus à penser au-dessus du peuple. Vatican II soumet ainsi l'institution sacerdotale à une conception ecclésiale qui la déborde toute part. À un tel changement de perspective, ou à la redécouverte d'une pratique antique, la forme politique du pouvoir moderne dans l'Église n'était pas prête. Elle s'est imposée à Vatican II parce qu'une bonne partie des évêques présents venaient de régions de mission ${ }^{15}$ où l'annonce de la foi devait être proche du peuple. L'Église se découvrait envoyée dans le monde avant d'être une entreprise qui a ses codes systématiques d'identification et d'opération. Ceux qui en restaient à une Église seulement institutionnelle étaient invités à une conversion nullement facile de leurs conceptions.

À la fin de Vatican II, certains disaient que le concile était allé trop loin, ou d'autres pas assez. L'Église en France a évidemment son histoire propre, où la laïcité et sa manière de sécularisation allaient bon train, entre autres après la seconde guerre mondiale durant laquelle les murs entre les croyants et les incroyants s'étaient fissurés, pour ne pas dire qu'ils étaient tombés. L'excellence humaine, et évangélique, n'est plus l'apanage du système ecclésial. On en vient alors à se dire que la hiérarchie n'est pas nécessaire pour vivre en chrétien. Roustang, attentif à ce qui se passait à l'époque dans son pays, note que, dans la communauté chrétienne, audelà de l'opposition des partis de la tradition ou de l'innovation, naissait un troisième homme, celui qui prend la parole avec liberté. L'article tente de décrire ce troisième homme, un chrétien qui a autre chose à penser qu'à une répétition liturgique et dogmatique, ou à tout chambouler. Le troisième homme ne s'intéresse pas à cela, mais à la vie sociale au milieu de laquelle il annonce la Bonne Nouvelle selon sa conscience.

Vatican II, puis Jean-Paul II, ont reconnu que l'Église peut se tromper. La presse mondiale a fait ses gros titres de ces aveux. Les affirmations où

\footnotetext{
${ }^{14}$ ROUSTANG, Fr. "Le troisième homme". In : Christus 13, 1966, pp. 561-567. Cet article a été édité de nouveau par ROUSTANG, E.-A. et al. (éd.). François Roustang : Le troisième homme, entre rupture personnelle et crise catholique. Paris : Odile Jacob, 2019, p. 19-27. Nous citons cette édition.

${ }^{15}$ N'oublions pas que le livre de GODIN, H. - DANIEL, Y. France, pays de mission. Paris : Cerf, est de 1943. Il est republié en format de poche en 1962, en plein concile.
} 
l'Église s'identifiait aux paroles du Christ ne sont plus à tenir à la lettre - c'est le moins qu'on puisse dire ${ }^{16}$. Les chrétiens sont dès lors invités à agir en conscience, et non plus en suivant quelque idéologie, peu importe qu'elle soit de droite ou de gauche. Un appel à la conscience personnelle résonne, qu'il n'est cependant pas facile à mettre en place. Le met en évidence la publication deux ans plus tard, en 1968, de l'encyclique Humani generis de Paul VI, et le refus de l'écouter de la part de nombreux couples de chrétiens engagés. Roustang anticipe en 1966 l'attitude de ces chrétiens, figures typiques $\mathrm{du}$ « troisième homme ».

L'exigence qui pèse sur eux est maintenant beaucoup plus grande, beaucoup plus profonde et vraie. Il ne s'agit plus d'obéir automatiquement à une loi, mais de comprendre et de respecter l'autre sans lui imposer un poids qu'il ne peut porter, en reconnaissant le désir de l'autre dans la lucide acceptation progressive du sien propre (22).

Vatican II avait ouvert des voies qui « ont réveillé la conscience chrétienne qui, au nom même de la foi, va beaucoup plus avant que ne le souhaitent les autorités » (22). Combien de valeurs chrétiennes ne sont-elles pas à reconnaître dans ce monde ? La santé publique n'est-elle pas parlante en termes de salut ? De là d'ailleurs l'écho mondial de documents pontificaux comme Pacem in terris de Jean XXIII, en 1963. L'Église sait toucher le cœur des gens, indépendamment de ses jargons théologiques, parce que l'attente de l'Évangile a déjà été semée en eux.

Le texte de Roustang a été republié en 2019 avec des commentaires d'historiens et de spécialistes en sciences humaines ${ }^{17}$. Cette nouvelle édition a été présentée à peine sortie de presse par l'actuel directeur de Christus, Remi de Maindreville. On ne peut pas nier que Roustang avait prévu les secousses qui allaient ébranler le peuple de Dieu et la hiérarchie de l'Église en France et ailleurs, parce qu'il avait annoncé " un troisième homme qui nous sort en vérité des impasses mortelles et appelle l'Église à s'ouvrir à des dimensions toujours plus grandes de Celui qui la fait vivre » (115). Nous ne nierons pas cependant que "le philosophe Roustang était davantage sensible à l'illusion d'une liberté sans ancrage ni réalité institutionnels » (115). De même, Ghislain Lafont, dans son Blog « Des moines et des hommes » du 16 avril 2019, salue la nouvelle édition du texte de Roustang, vieux de plus qu'un demi-siècle, en observant que l'Église était alors invitée à entrer dans une nouvelle époque avec " une vision plus profonde, c'est-à-dire plus divine et humaine à la fois, qu'on pourrait résumer par cette sentence : “Dieu est amour et il est permis d'être

\footnotetext{
${ }^{16}$ Notons cependant que, dans cette problématique, n'entre jamais en compte la tradition des quatre sens de l'Écriture (voir DE LUBAC, H. Exégèse médiévale : Les quatre sens de l'Écriture. Paris : Aubier, 1959-1964), et surtout des trois sens «spirituels» qui manifestent combien le sens « dogmatique » ne peut pas s'approprier de l'entièrement du discours chrétien.

${ }^{17}$ ROUSTANG, E.-A. et al. (éd.), François Roustang, p. 19-27.
} 
homme" ». Et le moine de la Pierre-qui-vire de citer Thomas d'Aquin (qui cite en fait Jean Damascène) pour confirmer ce qu'il vient de dire : cet homme, image de Dieu, est « principe de ses actes en tant qu'il a sur eux le libre arbitre et la puissance ${ }^{18}$. L'attention à la conscience et à ses dimensions de prise en charge personnelle entre ainsi au cœur de la vie du christianisme.

\subsection{Georges Morel}

François Roustang a dirigé avec Michel de Certeau la revue Christus jusqu'à la grande crise de $1966^{19}$. Georges Morel travaillait par contre aux Études que dirigeait Bruno Ribes et où arriva bientôt de Certeau. Les réflexions dans ces centres de culture chrétienne sont très pointues. 1966 est aussi l'année de publication des Mots et les choses de Michel Foucault. Que la raison ne soit pas immuable, qu'elle ait une histoire, et une histoire politique, voilà qui ressort d'arguments dont il est impossible d'ignorer les conclusions. La raison est historique. Les œuvres culturelles sont historiques. Le christianisme est dans l'histoire de la culture en Occident.

Le premier ouvrage de Morel, 3 volumes sur Jean de la Croix ${ }^{20}$, met au cœur de sa réflexion sur le langage mystique une perception de la subjectivité que les structures mentales de l'extrinsécisme, toujours présentes dans une théologie facile de l'immuable, ne parviennent pas à assumer. Selon l'auteur, comme pour de Certeau d'ailleurs, l'étude de la mystique permet d'élaborer une anthropologie où la finitude humaine n'est pas méconnue, ou effacée sinon anéantie dans ce que beaucoup imagine être une adhésion à Dieu mais qui ne serait autre qu'une divinisation imaginative de l'homme. L'homme mystique reste un homme fini, attentif cependant à dire ce qui n'est pas pour l'homme fini un enfermement carcéral. Le mystique vit du désir d'aller plus en avant dans des chemins inconnus dont il a un certain savoir sans pouvoir s'arrêter à quelques formes symboliques comme si cela lui suffisait.

La réflexion de Morel sur une anthropologie de l'homme fini se poursuit dans des articles publiés dans les Études entre 1964 et 1967. Le symbolisme est considéré celui-ci une "interface », dirait-on aujourd'hui, qui n'est aucun des pôles qui s'y rejoignent. L'anthropologie de la finitude met ainsi en crise les affirmations théologiques trop assurées d'elles-mêmes. Le chapitre final des Problèmes actuels de religion ${ }^{21}$, qui réunit quelques-uns de

${ }^{18}$ THOMAS D'AQUIN, Somme de théologie, Ia-IIae, Prologue.

${ }^{19}$ Voir FOUILLOUX, Ét. "L'affaire du « Troisième homme »". In : E.-A. Roustang et al. (éd.), François Roustang, pp. 31-33 : "Christus et Roustang ».

${ }^{20}$ MOREL, G. Le sens de l'existence selon saint Jean de la Croix. 3 vol. Paris : Aubier, 1970-1971.

${ }^{21}$ MOREL, G. Problèmes actuels de religion. Paris : Aubier-Montaigne, 1968. 
ces articles des Études, renvoie au christianisme, certes élément essentiel de la culture française, mais en attaquant l'idée de «Dieu » qui y serait trop souvent confuse.

Le livre trace d'abord à grand traits une histoire du christianisme en France. Le point de vue est sociologique (le christianisme n'a jamais connu d'âge d'or en Europe, quoi qu'on en dise, par exemple sur le Moyen-Âge) et philosophique (Marx, Nietzsche). Quand on parle d'athéisme ${ }^{22}$, on n'est donc pas plus au clair que quand on parle de christianisme. Il est important de repérer à quelle dimension humaine correspond celui-ci, à la problématique du sens, ou plus exactement du « sens du sens ». Dire que la vie humaine n'a pas de sens, c'est empiriquement trop souvent vrai, et pourtant la question du sens ne cesse de surgir dans la conscience humaine. «Il n'est pour l'homme qu'une possibilité humaine : le désir du sens pour le sens, le regard (re-spect) vers le sens pour le sens » (180), non pas pour le posséder ni pour s'y soumettre mais pour le chercher.

Plus profondément que les attitudes qui arrêtent le désir, le « sens » est une orientation que la conscience sait désirable, bonne et vraie, qui ne finit jamais à moi et ne désavoue jamais ma propre finitude. Morel reprend ainsi une définition de la « religion » qu'Emmanuel Lévinas avait proposée ${ }^{23}$ : «La société avec Dieu n'est pas une addition à Dieu ni un évanouissement de l'intervalle qui sépare Dieu de la créature. Par opposition à la totalisation, nous l'avons appelée religion » (183). Par principe en effet, "le rapport de l'homme à Dieu renvoie à un non rapport » (183). Je suis fini, mais je suis. En cela même, "nous savons avec de moins en moins de doute que notre existence d'être fini n'était pas nécessaire » (184).

Naît à ce moment en moi un sentiment nouveau, celui d'être libre, ou plutôt d'entrer « dans un univers qui est combat pour la liberté et où elle-même comme liberté se met en question » (184). Comment alors ne pas vivre une altérité radicale, et même originaire. La réflexion est très lévinasienne.

Ce que vise l'idée de relation sans relation, c'est un type de relation parfaitement original, plus exactement la relation-type, dont l'essence est de s'ouvrir, sans retour égoïste, à l'origine, conçue non d'abord comme origine mais en elle-même et pour elle-même. Et c'est par cette ouverture que se crée la communication, qui a ainsi pour forme et pour contenu le pur don (184-185).

Le mode de relation à l'origine pour elle-même ne sera-t-il pas dès lors autre que seulement moral, mais un mode de gratitude, d'action de grâce

\footnotetext{
${ }^{22}$ Il est bon de rappeler que, le 7 mai 1965, le pape Paul VI avait demandé aux jésuites, lors de la conclusion de la XXXIe Congrégation générale, de s'attacher en priorité à la lutte contre l'athéisme (voir les Décrets de la trente-et-unième Congrégation générale de la Compagnie de Jésus. Toulouse : Prière et Vie, 1965, p. 262-263).

${ }^{23}$ LÉVINAS, Em. Totalité et infini : Essai sur l'extériorité. Den Haag : Martin Nijhoff, 1990, p. 78-79 (texte cependant modifié dans la citation faite par Morel).
} 
et d'adoration ? « La véritable adoration est un acte réaliste et modeste. De toute façon, inchoatif »(206).

L'intention de Morel est riche d'un point de vue philosophique, et aussi partagé par de nombreux phénoménologues français. Notre connaissance de Dieu est un non savoir. Ce n'est pas là enfermer la raison dans l'ignorance, mais l'ouvrir à plus qu'au savoir selon la raison seule. Connaître Dieu, c'est le reconnaître - et l'adorer. Le reconnaître, requiert cependant un gros travail sur soi. Ce travail de reconnaissance, au sens d'action de grâce, offre enfin un savoir correct. " Penser quelque chose de Dieu, c'est en définitive penser à Dieu. Penser à lui comme lui » (211), sans l'enfermer dans quelque circuit de concepts préétablis. Morel cite à ce propos Jacques Derrida ${ }^{24}$ : «La paix comme le silence est la vocation étrange d'un langage appelé hors de soi par soi »(213), « un silence ouvert à la présence inépuisable, et source vive du langage » (214). Le «plus » que le langage indique est certes encore dans le langage mais pas comme n'importe quel langage. Il y a déjà dans nos langues des mots paradoxaux, qui disent ce qui ne peut jamais être représenté, le mot « infini » par exemple. Morel n'explicite pas cependant ce point. Ce serait sans doute risquer d'y perdre l'inquiétude sous les coups d'une explication qui espère en trouver une formulation dogmatique tranquillisante. Il se tourne plutôt vers la pratique du langage dans une communauté humaine où une multiplicité d'intentions de sens se noue nécessairement dans un accord spontané, ce qui est un véritable engendrement linguistique. En ce sens,

la théorie technique [qu'est la théologie] au service de la communauté ne peut que formuler ce souhait non platonique : qu'un jour tous les hommes aient la possibilité — et donc la liberté - de participer activement et directement (de moins en moins par procuration) aux doctrines qui essaient tant bien que mal d'exprimer le sens vécu et à vivre » (224).

N'est-ce pas cela, notons-le, qui devrait animer aujourd'hui l'idée de « synodes » diocésains?

Morel publie en 1970-1971 3 volumes sur Nietzsche, puis en 1976-1978, de nouveau 3 volumes intitulés Questions d'homme ${ }^{25}$. Le dernier de ces volumes $^{26}$, intitulé Jésus dans la théorie chrétienne, examine des données propres au christianisme. Des commentateurs jugent que ces pages manifestent que Morel a alors abandonné la foi chrétienne. Il prend en effet distance de la « théorie » chrétienne, c'est-à-dire de la théologie telle qu'elle a été formulée par les conciles des premiers siècles de l'ère chrétienne et sans doute simplifiée dans les catéchismes diocésains d'il y a peu de temps et que l'auteur

${ }^{24}$ Voir DERRIDA, J. L'écriture et la différence. Paris : Seuil, 1967, p. 172.

${ }^{25}$ MOREL, G. Nietzsche : Introduction à une première lecture. 3 vol. Paris : Aubier-Montaigne, 1970-1971. ; MOREL, G. Questions d'homme. 3 vol., Paris : Aubier-Montaigne, 1976-1978.

${ }^{26}$ Les deux premiers traitent des Conflits de la modernité et de L'autre. 
déclare "idéologique ». Le premier chapitre, " Du Jésus historique », fait siennes des questions souvent répétées aujourd'hui sur la véracité historique de nombreuses affirmations sur Jésus ou de Jésus. Le second démonte les « idéologies » qui soutiendraient la teneur théologique de ces affirmations. Le troisième se lance dans l'interprétation des évangiles pour y retrouver des points essentiels à retenir du point de vue du sens anthropologique. Le texte cite abondamment les Écritures en mettant en évidence l'humanité de Jésus que la théologie des miracles et d'autres actions merveilleuses écrasent sous une représentation causale de la divinité.

Les pages intitulées " De l'actualité et de l'inactualité de la Résurrection de Jésus » suffisent à faire voir comment procède Morel. Les évangiles racontent comment il fut difficile pour les disciples de Jésus de le reconnaître ressuscité. Parfois, ils ne le reconnaissent qu'au moment de sa disparition, comme ce fut le cas des disciples d'Emmaüs selon l'évangile de Luc. Les apparitions laissent souvent les disciples dans le doute : est-ce lui, ou bien qu'avons-nous vu ? Pour l'enseignement habituel du christianisme, les apparitions confirment que Jésus est ressuscité. Mais, selon Morel, ce n'est pas vraiment à cela qu'elles servent. À trop insister de cette façon, le croyant va rater ce dont il s'y agit. Le message vrai de la résurrection n'est pas celui de la confirmation de l'identité de Jésus ressuscité, mais celui de l'amour indéfectible de Dieu pour l'homme Jésus, à qui il donne sans cesse la vie, comme à tout homme. Les apparitions sont des témoignages de l'amour de Dieu, et n'ont pas de signification correcte en dehors de cela. On peut d'ailleurs soutenir que, dans le cas des disciples dont la culture spirituelle était plutôt fruste les récits évangéliques en donnent de nombreux témoignages - leurs récits ne pouvaient pas ne pas interpréter leurs expériences sans les détourner de leur sens vrai pour les adapter à leurs possibilités culturelles.

Nous avons là un cas de pédagogie générale de Jésus. Un signe évangélique ne vaut pas comme un effet physique dont on pourrait retrouver en amont la cause empirique. Dix versets après ces mots du discours du « pain de vie » : «si vous ne mangez pas la chair du Fils de l'homme et ne buvez pas son sang, vous n'aurez pas la vie en vous » (Jn 6,53), nous lisons : "C'est l'esprit qui vivifie, la chair ne sert de rien. Les paroles que je vous ai dites sont esprit, et elles sont vie » (Jn 6,63). La tension entre ces textes est forte. N'y aurait-il donc pas là, au cœur de la foi en l'incarnation, des risques d'équivoques ? " D'une part, [les disciples] croient dans une certaine mesure à l'amour et, d'autre part, ils ont besoin d'apparitions pour y croire » (203).

La position de Morel s'appuie ainsi sur une distinction entre

deux perspectives fondamentales : l'une, intérieure et l'autre, extérieure. L'élément invisible est en principe constitué par une foi remplie d'amour pour Jésus, disparu et vivant près du Père, et par une fraternité qui rend les chrétiens fils de ce Père. Et l'élément visible renvoie aux doctrines et 
aux pratiques. Les doctrines en effet s'élaborent [...]. Quant aux pratiques, il y a à l'intérieur de la communauté la prière toujours, la fraction du pain en mémoire du Disparu, la vie matérielle en commun (201-202).

La distinction de l'extérieur et de l'intérieur vaut aussi pour la résurrection : «Tout se passe dans les jours qui suivent la mort de Jésus comme si pour les disciples leur maître était devenu l'essentiel. Pour Jésus, la question n'est nullement celle de sa résurrection » (203). D'où la thèse : les apparitions " sont le contrecoup équivoque, mélange de force et de faiblesse, d'une expérience qui, si elle a sens, est d'un tout autre ordre » (204). "C'est à l'expérience fondamentale des disciples qu'il faut revenir : ils ont cru à la force d'un amour plus fort que la mort » (204). Là se trouve l'objectivité de leur expérience, qui précède d'ailleurs la mort de Jésus. L'amour d'abord, même si c'est en balbutiant. "Le problème n'est pas d'abord de chercher si oui ou non notre vie s'achève à la mort, mais comment aimer l'Amour sans anticiper à l'excès sur le chemin » (205).

Concluons cette section de notre article. Les auteurs que nous avons rencontrés mettent au centre de leurs préoccupations une antinomie évidente entre l'autonomie et l'hétéronomie. Pour Gauchet, l'histoire de l'Occident va de l'hétéronomie mythique à l'autonomie scientifique. Pour Roustang, le "troisième homme » prend en conscience la responsabilité de ses actes. Pour Morel, vivre de façon autonome signifie accepter sa finitude sans la fuir mais avec la conscience d'être aimé. Nous aboutissons ainsi à chaque fois à une spiritualisation du christianisme dans la conscience personnelle. Le propre du christianisme, par rapport aux religions autres que monothéistes, n'insiste-t-il pas pourtant sur l'importance du corps, des phénomènes, sur la "lettre »? Nous aurons à revenir sur ce point.

\section{Un christianisme réorienté}

L'antinomie de l'hétéronomie et de l'autonome a été au début du XXe siècle au cœur de la dispute théologico-philosophique sur le "modernisme ", un dérivé de la dispute des temps de la naissance du luthéranisme et du concile de Trente sur les relations entre la grâce et la nature. La revendication de l'autonomie de la liberté est cependant dépassée dans la philosophie récente, du moins chez certains philosophes qui, invités par Edmund Husserl, le fondateur de la phénoménologie contemporaine, s'attachent « aux choses mêmes $»^{27}$, ou regardent les réalités comme elles sont, c'est-à-dire tissées de multiples relations. La liberté est en relation ou elle n'est pas. Cette perspective soutient les réflexions des auteurs dont nous lirons maintenant quelques textes, Luc Ferry, Jacques Derrida et Maurice Bellet.

${ }^{27}$ HUSSERL, Ed. Recherches logiques, t. 2, Recherches sur la phénoménologie et la théorie de la connaissance. Première partie. Recherches I et II. Paris, PUF, 1996², p. 6. 
Déclarer sans nuance que la théologie n'est que théorie soumise à des idéologies est sans aucun doute violent. En réalité, il n'y a pas qu'» une » théologie. Elles sont même en grand nombre. Morel n'était pas ignorant en la matière. Pourquoi donc a-t-il exagéré à ce point, comme beaucoup d'autres auteurs «qui n'y croient plus », en schématisant son adversaire, l'autorité dogmatique, sinon pour simplifier l'originalité de sa proposition d'une herméneutique originale des récits évangéliques, par exemple de la résurrection du Crucifié. L'interprétation de Morel, qui est loin d'être sans intérêt, ne manque-t-elle pas d'être également "idéologique » ? Sa distinction entre l'invisible et le visible, même si elle semble soutenue par le titre du grand libre posthume de Maurice Merleau-Ponty, Le visible et l'invisible ${ }^{28}$, est-elle vraiment soutenable, surtout si elle ignore le chiasme du professeur du Collège de France, le « et » entre visible « et » invisible? On a vu aussi que la question du "sens », du "sens du sens », était au cœur des inquiétudes des auteurs que nous avons cités. Nous allons voir maintenant, précisément à partir de la question du sens, qu'est possible un autre regard sur le christianisme que celui des auteurs déjà mentionnés.

\subsection{Luc Ferry}

Il est largement accepté de dire qu'une religion correspond à une recherche du sens de l'existence, du bien qu'on y fait volontiers et du mal qu'on y subit malgré soi. Distinguons cependant dès maintenant les deux mots « sens » et « signification ». La « signification » est une détermination, dans un jeu linguistique donné, qui vient des définitions données dans un dictionnaire où elles se renvoient inévitablement les unes aux autres. Le monde de la "signification » est un monde cohérent et fermé. La parole « sens » a par contre une fonction d'ouverture, d'orientation, comme quand on parle de "sens unique ». De soi la signification est définie, tandis que le sens est indéfini.

La culture contemporaine, tout comme la scolastique rationaliste encore enseignée dans de nombreuses institutions de formation ecclésiastique, est friande de significations. Un système de signification est en effet indispensable pour mener à bien des projets, pour les réaliser dans un monde qui rejette a priori toute incohérence. Le sens a par contre du souffle, du charme artistique. Il libère des systèmes clos. Il nous transporte dans un monde de possibilités aventureuses. Il est aussi risqué, parce que nous ne pouvons pas lui attribuer des significations qui pourraient baliser de manière assurée ses chemins d'aventures. Sa finalité ne fait pas système, elle est gracieuse, ou gratuite. La culture d'aujourd'hui, qui n'a pas de grandes visées, le cadre dans l'immédiatement faisable. Par exemple, ten-

${ }^{28}$ MERLEAU-PONTY, M. Le visible et l'invisible. Paris : Gallimard, 1964. 
tés par le juridisme, nous voulons nous protéger de tous les côtés, nous assurer contre toutes sortes de faux pas commis ou subis. Ce faisant, nous enfonçons le sens réel sous la signification formelle.

Luc Ferry ${ }^{29}$ dénonce avec pertinence ce glissement lorsqu'il écrit ceci:

À l'intérieur de ces petits desseins qui sont comme autant de bulles closes sur elles-mêmes, nos actions prennent donc un sens : elles sont tout à la fois orientées dans une certaine direction et animées d'intentions qui leur confèrent, à nos yeux comme à ceux des autres, une certaine signification. Pourtant, la question du sens de ces projets qui donnent sens nous échappe. Dans la vie quotidienne, nous savons sans doute, à chaque instant ou presque pourquoi il nous faut accomplir telle ou telle tâche supposée "utile ", mais l'utilité de cette utilité demeure le plus souvent, lorsqu'il nous arrive d'y réfléchir, opaque et douteuse. Le « sens du sens » - la signification ultime de toutes ces significations particulières - nous fait défaut (15-16).

L'horizon de l'homme contemporain est devenu l'immédiat. Les distances spatiales n'existent plus, ni les temporelles. Un auteur italien a intitulé un de ses livres en lui donnant ce titre : Où es-tu ? Ontologie du téléphone portable ${ }^{30}$. Nos smartphones ont annulé les distances physiques. Quant au temps, il n'a plus de poids. Les prévisions en économie sont abandonnées aux statisticiens dont les annonces changent d'un jour au lendemain. Le passé est " passé », tenu sous silence ; les cours d'histoire sont de plus en plus ôtés des programmes des lycées. La fidélité des relations humaines, mises sous pression par la mobilité du travail, est réduite au «like » ou à son contraire. Ce n'est pas sans raison que Ferry reprend la formule de Weber et de Gauchet en rappelant l'exigence qui nous incombe aujourd'hui : "Le réenchantement de la politique : enraciner Dikè dans Philia. Retrouver du sens » (164) pour notre maison commune. Le travail des philosophes n'est-il pas de renouveler le sens du sens ? "Le vrai défi n'est pas que théorique, mais tout à la fois pratique et spirituel [...]. C'est dans Philia qu'aujourd'hui se décide au premier chef le sens de nos vies » (165). Puisse notre humanité globalisée retrouver le sens dynamique de nos affections.

\subsection{Jacques Derrida}

Les auteurs dont nous avons parlé dans la deuxième section de notre article opposaient idéologie et conscience (Roustang), visibilité et invisibilité (Morel). Mais ces oppositions, typées en extériorité et intériorité, sont-elles tenables ? La vie n'est-elle pas complexe, à la fois raison systématique et action aventureuse, connaissance et valeur ? Jacques Derrida, juif non

${ }^{29}$ FERRY, L. L'homme-Dieu, ou le sens de la vie. Paris : Grasset, 1996, p. 15-16.

${ }^{30}$ Voir FERRARIS, M. Dove sei ? Ontologia del telefonino. Milano : Bompiani, 2005. 
pratiquant, a été un maître dans la composition de plusieurs principes les uns avec les autres, jusqu'à d'ailleurs rendre son style difficile à suivre, virevoltant. Il a ainsi écrit un texte original sur la religion à l'occasion d'un colloque entre amis philosophes (Gianni Vattimo, Hans-Georg Gadamer, d'autres encore) à Capri en 1994. Son texte a été amplifié et publié peu après la rencontre sous le titre "Foi et savoir ${ }^{31}$.

Derrida démarre en désignant deux caractéristiques essentielles de la religion. La première : « le messianique, ou la messianité sans messianisme » (27). Dans le messianique, il y a une « ouverture à l'avenir ou à la venue de l'autre comme avènement de la justice, mais sans horizon d'attente et sans préfiguration prophétique » (27). Une thèse fondamentale pour Derrida s'impose ici, à la suite de Lévinas. "La venue de l'autre ne peut surgir comme un événement singulier que là où aucune anticipation ne voit venir, là où l'autre et la mort - et le mal radical - peuvent surprendre à tout instant » (27). De là cette foi en l'impossible qu'on retrouve dans la religion, qui n'est pas un savoir mais un "invincible désir de justice » (28), lequel « s'inscrit d'avance dans la promesse, dans l'acte de foi ou dans l'appel à la foi qui habite tout acte de langage et toute adresse à l'autre » (28). Derrida évoque par là une forme universelle de la religion qui, pour une raison transcendantale, est intrinsèque à tout acte humain, y compris à la science. « Le mystique ainsi entendu allie la croyance et le crédit, le fiduciaire ou le fiable, le secret [...] au fondement, au savoir » (29.

La première caractéristique de la religion s'appuie aussi sur la tradition philosophique platonicienne, sur $\chi \omega \varrho \alpha$, un terme qui vient du Timée de Platon $^{32}$ et dont l'horizon est le passé, non plus le futur du désir. La $\chi \omega \varrho \alpha$ signifie un "rien originaire ", moins encore que le tohubohu de la Genèse, peut-être ce fond sans fond que Heidegger évoquait dans son Introduction à la métaphysique ${ }^{33}$. Platon ne la nomme que de manière métaphorique, comme une matrice par exemple. On la traduit suivent par " matière », ce qui serait ambigu. Nous sommes là en réalité dans la tradition de l'origine indicible, devant laquelle la raison perd toute assurance en soi et dont il convient de maintenir les droits. Les efforts de la raison à vouloir tout saisir manifeste cette impossibilité qui lui est intrinsèque, qu'elle cache en soi sous des formes nobles mais édulcorées, dialectiques, analogiques, en moment négatif de la réflexion, en interdiction de connaître mais en obligation de penser. La religion rend ainsi attentif à un futur comme à un passé l'un et l'autre inaccessibles, hors de portée.

La seconde caractéristique s'oppose d'une certaine manière à la première. La religion est en effet aussi solide, immuable, « indemne ». Elle requiert

\footnotetext{
${ }^{31}$ DERRIDA, J. "Foi et savoir". In : J. DERRIDA - G. VATTIMO, La religion, Paris : Seuil, 1996, p. 9-86.

${ }^{32}$ PLATON, Timée, 52a8.

${ }^{33}$ HEIDEGGER, M. Introduction à la métaphysique. Paris : Gallimard, 1967, p. 15.
} 
une attitude de "fiance, fidélité, confiance, foi », de désirs possibles qui naissent en moi, une " expérience de la sacralité, voire de la sainteté, de l'indemne sain et sauf » (49) qui s'impose à moi.

Le discours religieux tourne nécessairement autour de ces deux foyers en ellipse, l'invisible et le visible, l'indécidable et le décidé, le désir d'attention confiante et l'imposition de l'origine, « le respect, la patience, voire la pudeur ou la piété » (50) et en même temps un lien " à l'obligation, au ligament, donc au devoir et donc à la dette » (51), au sein « d'une division de la source et du sens » (51). La forme d'un discours sur la religion ne peut donc pas être circulaire mais elliptique, foi et raison, l'une s'invitant dans l'autre, se limitant et se stimulant mutuellement, sans qu'aucun discours ne puisse prendre possession de l'autre, s'auto-définir pour limiter l'autre, s'en tenir au large. L'auteur reprend alors un balancement que Étienne Gilson voyait chez Thomas d'Aquin entre le révélé et le révélable ${ }^{34}$. Le révélé est une donnée, le révélable une possibilité issue de l'inaccessible. « Le respect de cette indécision singulière ou de cette surenchère hyperbolique entre deux originarités, entre deux sources, entre, disons par économie indicative, l'ordre du "révélé" et l'ordre du "révélable", n'est-ce pas à la fois la chance de toute décision responsable et d'une autre "foi réfléchissante", d'une nouvelle "tolérance" » (32) ?

\subsection{Maurice Bellet}

Derrida invite à ne pas durcir nos discours en oppositions par principe irréconciliables. Roustang et Morel n'ont-ils pas succombé à ce péril ? Dans sa présentation de la nouvelle édition de l'article de Roustang sur « Le troisième homme », de Maindeville renvoie à Maurice Bellet, qui «passera vingt ans et quatre-vingt articles à aider les lecteurs à se situer dans une foi intelligente et vivante au sein d'un monde devenu évolutif $»^{35}$. En 1965, Bellet publie Ceux qui perdent le foi dans la collection Christus dirigée par Roustang $^{36}$. Ce n'est pas là un ouvrage d'apologétique classique car, insiste l'auteur, les mots du titre sont beaucoup moins clairs qu'en apparence. Il s'agit plutôt de reconnaître que ce qu'indiquent ces mots ne nous est pas extérieur, comme des faits étrangers, mais est aussi en nous, chrétiens, croyants confirmés et boiteux, et que le premier pas est de le reconnaître.

\footnotetext{
${ }^{34}$ GILSON, Ét. Le thomisme : Introduction à la philosophie de saint Thomas d'Aquin. Paris : Vrin, $1944^{5}$, p. 20.

${ }^{35}$ DE MAINDREVILLE, A. "François Roustang : Le troisième homme". In : Christus avril 2019 , p. 115.

${ }^{36}$ BELLET, M. Ceux qui perdent le foi. Paris : Desclée de Brouwer, 1965. Voir LANGLOIS, Cl. "Un long compagnonnage. François Roustang et Michel de Certeau à Christus". In : E.A. ROUSTANG et al. (éd.), François Roustang, p. 63-98, surtout 84-87 ("Maurice Bellet, le troisième homme de Christus").
} 
Ceux qui «perdent la foi » nous sont proches, nous provoquent intimement pour que nos certitudes perdent leurs prétentions rationnelles et s'approfondissent en dimensions humaines.

La vie fait en effet que des discours de la foi peuvent perdre leur sens ou leur fonction de repérage, devenir plus obscurs pour une intelligence qui devrait féconder nos actions et leur donner la vie. Soyons honnêtes. Voilà pourquoi

nous espérons éviter d'aborder abstraitement «l'incroyant », nous tenterons de demeurer à l'intérieur d'une relation humaine effective, de ne jamais planer au-dessus, comme si nous étions Dieu même, qui fait pleuvoir sur les justes et sur les autres, et embrasse d'un seul regard tous les hommes. Nous sommes ce que nous sommes, et rien de plus; et pourtant, nous portons Dieu (15).

En conclusion de son ouvrage, l'auteur indique un programme à réaliser :

La foi est-elle en nous principe d'une méthode qui nous fait découvrir sa région dans la vie sociale des hommes ? Si ce n'est pas cela que notre pensée chrétienne aborde, en tant que tel, est-ce que l'effort sociologique des chrétiens ne reste pas en marge de ce qui lui est essentiel, ou condamné à y entrer avec des instruments de pensée équivoques ? (160)

La tâche proposée est originale, exigeante. Elle invite à reconnaître d'abord que le système dogmatique du christianisme ne dit rien à la vie de foi dans nos sociétés réelles, alors que la foi pourrait au contraire y créer des espaces et des temps de recréation.

Si nous discernons les exigences d'une parole vraie, nous sommes à même de nous engager dans la réexpression, le dévoilement de l'actuel, la construction du langage, etc. Mais tout cela est à faire (160-161).

Les années de ce livre de Bellet sont celles de la démythisation, dont témoigne par exemple les Colloques Castelli à l'université La Sapienza de Rome ${ }^{37}$, où sont intervenus de auteurs francophones de la taille de Stanislas Breton, Claude Bruaire, Alphonse de Waelhens, Gaston Fessard, Henri Gouhier, Emmanuel Lévinas, Paul Ricœur, Xavier Tilliette, Antoine Vergote, etc. Le livre de Ricœur sur Freud est aussi de $1965^{38}$. Bellet, appelé en 1964 par Roustang à Christus, l'accompagne-t-il ensuite dans ses aventures psychanalytiques ? En 1979, il publie Le Dieu pervers ${ }^{39}$, où est dénoncé le discours de mentalité augustinienne habituelle dans l'Église, qui exile la sexualité, ou le corps en général, d'une vision intégrative ou systémique de la personne humaine. Pourtant, la réflexion sur le corps

${ }^{37}$ CASTELLI, E. (éd.). Démythisation et morale. In : Archivio di filosofia, 1965. ; CASTELLI, E. (éd.). Démythisation et idéologie. In : Archivio di filosofia, 1973.

${ }^{38}$ RICCEUR, P. De l'interprétation : Essai sur Freud. Paris : Seuil, 1965.

${ }^{39}$ BELLET, M. Le Dieu pervers. Paris : Desclée de Brouwer, 1979. 
a pris une extension nouvelle durant la seconde moitié du XXe siècle, surtout dans la mouvance de la phénoménologie. Le livre Philosophie et phénoménologie du corps, de Michel Henry, est de 1965 ; la Philosophie du corps de Claude Bruaire est de $1968^{40}$. La théologie pourrait en tirer profit, ainsi que sa «traduction » dans les homélies destinées au grand public.

Les interrogations sont vives. Le discours officiel de l'Église ne déplace ses paradigmes culturels que lentement. La critique de Bellet souligne l'incompréhension que laisse encore l'annonce du message chrétien. Le lexique des autorités du corps ecclésial est aujourd'hui en train de perdre du sens auprès des chrétiens, qui donc quittent l'Église. Un exemple, dramatique : en 1968, Paul VI publie l'encyclique Humanae vitae, dont la doctrine sur la contraception heurte beaucoup de chrétiens engagés par ailleurs dans le témoignage de la foi. La révolution sexuelle de la culture contemporaine touche certes au cœur les énoncés doctrinaux chrétiens, et on ne voit pas comment l'affronter sans s'y opposer massivement. Les catégories phénoménologiques du corps et de la chair ne pourraient-elles pas y aider ?

En 2001, Bellet publie La quatrième hypothèse ${ }^{41}$. L'auteur décide de ne pas considérer trois hypothèses : 1 . que le christianisme disparaisse en se sécularisant de lui-même ; 2 . qu'au contraire le siècle aspire en soi ses valeurs ; 3 . que, malgré tout, le christianisme maintienne ses traditions rituelles et rationnelles comme si de rien n'était. La quatrième hypothèse, fidèle au livre de 1965, invite à " radicaliser les exigences de l'écoute et de la liberté » (6) afin d'y découvrir des espaces d'attente de Dieu. " La force de l'Évangile [...], c'est d'annoncer que le chemin de chacun est son chemin, parce que c'est à lui que la parole est adressée » (31). N'avonsnous pas des idées formées a priori sur le cœur et l'intelligence du peuple chrétien que touche, pourtant mais autrement, la Parole libre de Dieu ? Ce sera là où elle est accueillie, chez ceux qui, sans taire leur voix humaine, deviennent «témoins vivants » (31) malgré toutes les limitations. La foi chrétienne est paradoxale. Pilate le révèle : «Voici l'homme » (Jn 19,5), parole prophétique qui annonce le commencement d'une humanité nouvelle, du témoin vivant, à travers l'effondrement de ses formes humaines communes et la destruction de sa parole originaire.

N'est-ce pas parce que les institutions religieuses ignorent la vérité évangélique du triduum pascal que l'homme en perd le sens ? Voici l'homme, l'être homme, simplement, le destinataire du choix divin, l'élu. Selon la quatrième hypothèse,

le vieux schéma religieux éclate, la religion n'est plus où elle est, il s'agit d'autre chose, que nous ne savons même pas nommer, que nous pouvons

\footnotetext{
${ }^{40}$ HENRY, M. Philosophie et phénoménologie du corps. Paris : PUF, 1965 ; BRUAIRE, Cl. Philosophie du corps. Paris : Seuil, 1968.

${ }^{41}$ BELLET, M. La quatrième hypothèse : Sur l'avenir du christianisme. Paris : Desclée de Brouwer, 2001.
} 
évoquer quand même, en parlant de naissance d'humanité, d'assurance primordiale, d'ouverture de la vie possible, d'émergence hors de la destruction (93).

Bellet met alors la pression : la communauté humaine, de l'Homme, est une communauté d'écoute mutuelle, d'attention d'homme à homme, sans autre exclusion que d'exclure car nul ne sait ce qui, en chacun, est témoin $\mathrm{du}$ « reste d'Israël ». Écouter, espérer, attendre.

Il semble, alors, que se répète quelque chose de ce qui avait paru, au cœur et à la pointe de l'effondrement : il faut sortir, il faut se tenir hors du christianisme, pour que ce que le Christ apportait au monde vienne enfin en toute sa force : un universel de l'écoute et de la convivialité, qui touche par en bas et par en haut à l'extrême de l'homme, qui ne tient pas par une idée, une doctrine, une culture, une politique, mais par l'homme même; dans le déchirement irréparable de tout ce qui assure l'empire sur les humains. Cassure du cercle, de ce grand cercle où sont clos la politique, l'économie, la culture même ; et qui se manifeste par la répétition inlassable, - hélas — des mêmes procédures d'exploitation, d'exclusion, de persécution (94).

\section{La Parole évangélique}

éveille l'Esprit (l'Esprit Saint!) présent en celui qui écoute. Alors l'écoute de la parole entendue coïncide avec l'écoute intérieure - celle qui éveille en l'homme sa vie, qui lui donne de franchir le pas qui le sépare du mortifère. [...] C'est pourquoi le Christ n'y est pas historique ou dogmatique, ou image pour la piété. Il est l'humanité et le tout (102).

Dans la troisième section de notre article, nous avons rencontré trois auteurs qui ne se contentent pas de contester la pertinence des discours théologiques, ce qui était la tendance des auteurs lus dans la deuxième section, mais qui invitent à recréer un langage qui ait un sens pour aujourd'hui. Cela ne se fera pas à partir de la seule expérience de l'autonomie subjective, de la conscience d'être responsable de soi et de ses conditions dans la finitude. Il faudra plutôt tenir compte de ce que à quoi la philosophie contemporaine s'est rendue attentive, à l'altérité, à l'autre, à l'infiniment distant. Aucun discours n'est autosuffisant.

Découvrir que le sens a un sens, comme le rappelait Luc Ferry, est une exigence très humaine car il n'est jamais immédiatement disponible sinon dans sa recherche même. Beaucoup n'y prête pas attention, mais le propre du langage humain est de ne pas s'enfermer dans son « dit », mais de se laisser dynamiser par son « dire » dont l'origine et la fin ne sont jamais arrêtées. Le cercle herméneutique ne suffit pas pour comprendre la religion, qui est à la fois messianique et insaisissable, disait Jacques Derrida. Le désir qui s'y exerce ne se nourrit pas de sa propre tension. La tension de l'esprit est déjà une réponse à un appel. Elle ne supplante jamais l'appel et ses mots n'y correspondent jamais adéquatement. La réflexion de Maurice Bellet sur l'Ecce homo invite enfin à revenir à la lecture de l'Évangile, à 
le lire en nous laissant toucher, car seulement ainsi l'Évangile nous parle et nous meut. À cette condition, le langage théologique se renouvellera. L'Évangile est vivant dans ceux qui l'accueillent et se laissent transformer par lui, leur donnant des mots nouveaux mais compréhensibles, leurs pensées en s'engageant dans une conversion radicale, un retournement de tout l'être. Ce n'est qu'alors qu'un langage sera théologiquement juste parce que structuré intérieurement par l'écoute du Verbe et de l'Esprit, et la disponibilité à se mettre en marche sur des chemins de résurrection que nous n'aurions jamais pu découvrir de nous-mêmes.

\section{Le christianisme à venir}

La crise du christianisme mise en avant et aussi en scène par les auteurs que nous avons mentionnés impose maintenant de préciser comment entendre l'expression «à venir ». En général, le mot « avenir » signifie ce qui va arriver, qu'on peut espérer ou craindre. Ceux qui aiment l'Église et qui prennent leur responsabilité dans l'annonce de l'Évangile peuvent être pris d'angoisse à considérer les statistiques de la pratique religieuse, c'est-à-dire essentiellement la participation aux rites du christianisme, y compris à la prière commune et à l'écoute des Écritures. D'un point de vue quantitatif, il est juste de se préoccuper de ce que deviendra sociologiquement le christianisme. Les énergies «fossiles » offrent une bonne métaphore de ce christianisme : elles ont chauffé et illuminé nos cités, mais maintenant elles arrivent à leur extinction, et nous n'avons pas fait tout le nécessaire pour les renouveler. Les rites et pratiques se sont sclérosés et n'ont plus de mots pour dire leur sens vital dans le monde d'aujourd'hui qui va de l'avant sans s'en préoccuper. On n'y trouve plus de signes qui soient signifiants pour le quotidien commun. Il y a un décalage énorme entre les pratiques de la foi et l'évidence de leur sens.

Le christianisme a été juif et grec, puis enrobé dans le latin. Chaque fois, les intellectuels y mettaient la marque sur leur culture maternelle ou empruntée. Les sources du christianisme ont ainsi été modifiées, selon les cultures qui les accueillaient. Le Moyen-Âge a jugé que le christianisme était digne d'un discours savant, scientifique selon les normes d'alors ${ }^{42}$. Avec la Modernité, la science a pris son autonomie et le langage chrétien s'est retrouvé écarté de la créativité culturelle. Les clercs sont restés des penseurs à la traîne. Ils ont été remplacés par les découvreurs laïcs des milieux intellectuels et puissants d'un monde libre de chercher et de penser. Le christianisme n'est plus créatif depuis longtemps mais réactif

${ }^{42}$ Voir CHENU, M.-D. La théologie au douzième siècle. Paris : Vrin, 1957, p. 323-350 : “Les Magistri. La « science » théologique". 
aux cultures nouvelles, sans voir comment participer à leur déploiement ${ }^{43}$, le plus souvent en résistant. Suivre une culture impose d'en adopter les paradigmes, ce qui éveille des débats inévitables dans tout groupe humain rassemblé autour d'une idéologie traditionaliste ou innovante. Les difficultés rencontrées par les théologiens au temps de Galilée ne sont plus à rappeler. L'autoréférentialité des discours ecclésiastiques, dont le dogme de l'infaillibilité pontificale est la manifestation la plus splendide ${ }^{44}$ et très mal expliquée, a conduit à écarter le discours catholique hors de la culture des sciences dont l'utilité et les progrès sont indéniables.

La constitution de Vatican II sur la liturgie, Sacrosanctum concilium, promulguée en décembre 1963, invite au n. 54 à célébrer les sacrements dans la langue utilisée couramment par les participants. Premier texte voté par les pères conciliaires, il a engagé un processus qui a troublé profondément la vie chrétienne. Des groupes de laïcs se sont constitués pour étudier les Écritures, l'histoire de l'Église, etc., et ils ont trouvé un langage dont l'étrangeté devenait évidente. L'humanité a été rendue au Christ et au christianisme, après des siècles de rationalisation de la foi. "Voici l'homme ».

À parler de l'avenir du christianisme d'un point de vue historique, c'est-à-dire dans la perspective d'un futur sociologique, nous ne pouvons pas édulcorer ce que nous venons d'affirmer et ne pas nous inquiéter du sens des mots où s'exprime le christianisme. Une autre entende du mot " avenir » est cependant possible, le christianisme " qui vient», une expression qui fait écho au « Viens, Seigneur Jésus » de l'Apocalypse $(22,17)$, un «Viens » qui est du Seigneur qui vient " maintenant», non seulement de demain. "Que l'homme assoiffé s'approche, que l'homme de désir reçoive l'eau de la vie, gratuitement », dit encore l'A pocalypse. La philosophie contemporaine peut aider à entendre cette actualité de la venue du Seigneur, particulièrement dans sa mouvance existentielle et phénoménologique.

Les auteurs que nous avons mentionnés sont largement sous l'influence de thèmes contemporains en philosophie tels que la finitude et l'altérité. La catégorie de la finitude est mise au cœur de la pensée depuis le milieu du XXe siècle, depuis une crise de conscience qui entend ne plus se laisser prendre par des fantasmes de puissances techniques adaptes à captiver l'imagination facile et médiatique ${ }^{45}$. La bombe atomique a donné un coup de fouet à la recherche scientifique, en consentant à combien de morts ? L'humanité ne pouvait-elle trouver que là de quoi assouvir sa soif indé-

\footnotetext{
${ }^{43}$ Cette affirmation devrait toutefois être nuancée : l'éducation des jeunes, conditions majeures pour le devenir des sciences, était assurée en grande partie par des clercs.

${ }^{44}$ On notera cependant que les pères conciliaires ont prudemment déterminé des conditions strictes de l'exercice de ce privilège pontifical, si bien qu'il n'a guère été mis en œuvre en dehors de décisions de dévotion.

${ }^{45}$ Voir HUSSERL, Ed. La crise de l'humanité européenne et la philosophie. trad. N. Paris : Hatier, 1992.
} 
finie d'énergie ? L'existence humaine est finie, la mort est un fait sérieux, soulignait Søren Kierkegaard ${ }^{46}$. Le christianisme a certainement à dire à ce sujet, à condition toutefois de ne pas parler de la résurrection comme si la mort n'était qu'un mauvais moment à traverser après lequel tout serait à peu près comme avant, c'est-à-dire à condition de repenser les expressions homilétiques qui parlent de manière trop facile de la résurrection et de retrouvailles post-mortelles.

Stanislas Breton, prêtre passioniste, a écrit parmi ses derniers livres un texte intitulé L'avenir du Christianisme ${ }^{47}$. Il commence son ouvrage par un chapitre sur la théologie de la Croix, sur la "Kénose » du Christ comme on le dit après saint Paul ( $\mathrm{Ph} \mathrm{2,7),} \mathrm{que} \mathrm{des} \mathrm{théologiens} \mathrm{modernes} \mathrm{dis-}$ tinguent de la théologie de la gloire. Un prêtre passioniste comme Breton ne pouvait pas, évidemment, se contenter d'une telle opposition. Avec le terme "kénose », "il ne s'agit pas [...] d'une simple vacuité de fait, ou d'un état de vide selon le langage courant, mais d'un acte en sa radicalité de parfait évidement, ou renoncement à tout ce que l'on est et à tout ce que l'on a » (20). Tout amour se donne, désire honorer son destinataire, se rendre semblable à lui. Dans la foulée d'une réflexion qui culmine dans l'amour, la phénoménologie récente, surtout avec Jean-Luc Marion ${ }^{48}$, a mis en avant l'importance philosophique de la catégorie du « don ». Breton connaît la tradition de cette catégorie.

"Les plus beaux de nos dons implorent la pitié d'une transcendante miséricorde. Ils n'en sont pas moins inspirés par l'Ineffable qui habite en nos cœurs » (23). La kénose ou l'abaissement jusqu'à l'être simplement homme, n'est pas une négation de la divinité de Jésus, puisqu'elle manifeste au contraire la liberté du don divin, Dieu libre de soi à nul autre pareil. D'un point de vue simplement anthropologique, «c'est par la médiation du don que l'esprit accède à la personnalité. En effet, la donation se dissocie mal d'un visage et d'une main. Le don ne cesse d'évoquer le donateur » $(39)^{49}$, et donc une reconnaissance. Certes, la circularité du don et du contre-don, du donateur et du donataire, est critiquable. Elle a habituellement un aspect commercial : à qui « donne » de l'argent le commerçant « donne » une marchandise. Derrida s'est employé à dénoncer ce $\operatorname{commerce}^{50}$, et Marion

${ }^{46}$ KIERKEGAARD, S. "Sur une tombe". In : ID., CEuvres complètes, t. VIII, Paris : L'Orante, 1979, pp. 61-89. "Car la mort même comporte un séreux qui ne tient pas à l'événement, au fait extérieur [...]. La mort justement peut apprendre que le sérieux réside dans l'intérieur » (p. 63).

${ }_{47}$ BRETON, St. L'avenir du christianisme. Paris : Desclée de Brouwer, 1999.

${ }^{48}$ Voir MARION, J.-L. Étant donné : Essai d'une phénoménologie de la donation. Paris : PUF, 1997.

${ }^{49}$ La lecture théologique du donateur a ouvert un débat passionné engagé par JANICAUD, D. Le tournant théologique de la phénoménologie française. Combas : L'Éclat, 1991. Nous n'y insisterons pas ici.

${ }^{50}$ DERRIDA, J. Donner la mort, t. 1, La fausse monnaie. Paris, Galilée, 1991. 
aussi mais en répondant à la critique de Derrida ${ }^{51}$. Le donateur peut par exemple faire un don anonyme à une ONG ou une ASBL : son présent sera sans sa présence, c'est-à-dire sans qu'il soit reconnu. Le don de Dieu sous la forme de la kénose n'impose d'aucune manière sa présence glorieuse ; et qui la reconnaît peut même ne pas y correspondre. L'action de grâce n'est pas de l'ordre de la nécessité. La liberté de celui qui accueille un tel don n'est d'aucune façon obligée, mais sa réponse révèle une potentialité ou un désir proprement humain.

Le passage par la Croix est de cet ordre. L'expression « chemin de croix » est riche de sens. Un chemin est destiné à porter vers un lieu. Le discours qui l'exprime s'appelle "odologie ", qui médite sur un éloignement à parcourir en marchant. Dans l'évangile de Jean, les disciples demandent à Jésus : «Où demeures-tu ? » (Jn 1,38). Et Jésus de répondre : «Venez et voyez ». La demeure n'est-elle pas elle-même une marche, sans doute jusqu'à Jérusalem ? Être dans la demeure, c'est agir. "L'agir est plus radical que le faire » (100) qu'il accompagne en lui donnant une saveur d'engagement spirituelle. "Grâce à cet esprit qui accompagne toute action, l'âme n'est jamais esclave de ce qu'elle fait, car elle a en elle le référent "céleste" qui empêche de "coller aux choses" ou d'en épouser la fascination » (101).

Selon Breton, les sagesses orientales peuvent inspirer les chrétiens. Elles connaissent ce chemin, qui est un passage vers les profondeurs humaines. "La vie profonde, qui coïncide avec l'agir en tant que "demeurer" dans le principe, se situe au-delà des questions pratiques de choix entre ceci ou cela, entre faire et ne pas faire » (108). La demeure est un chemin. Cheminer dans cette demeure, n'est-ce pas passer « du sommeil où s'attarde le vieil homme au matin du monde et de l'homme nouveau ? Passage difficile qui requiert abnégation et mort à soi-même » (108) : on aura reconnu ici le passage et la Pâque du Seigneur.

La réflexion sur ce passage, qui appartient aussi à tout désir, ne se contente pas d'opposer les catégories mort et vie, absence et présence. Breton n'apprécie pas l'opposition de catégories qui renvoient les unes aux autres en un balancement dialectique et indéfini. Son ouvrage considère en effet des catégories que nous rencontrons souvent en opposition, par exemple celles de la foi et de la sagesse dans le chapitre 2 de la première épitre aux Corinthiens. Les textes que nous venons de citer valent en effet pour la foi autant que pour la sagesse. Toutes désirent la profondeur de l'engagement de soi. Mais pour la religion ? Breton n'hésite pas à définir le christianisme par un contenu doctrinal, notionnel ou théorique. D'ailleurs, qu'entendre par « religion »?

Quand nous parlons de religion, il s'agit toujours d'un monothéisme fondé lui-même sur une parole originelle qui est parole de révélation. Cette parole

${ }^{51}$ J.-L. MARION, Étant donné, p. 108-121. 
fonde une communauté de fidèles, liée à une liturgie sacrificielle et à un ensemble de prescriptions ou de pratiques dont relève, parfois jusqu'au détail alimentaire, l'existence quotidienne (58).

La religion a donc une visibilité sociale. Elle supporte aussi un ensemble d'affirmations. Dans le cas du christianisme, ces affirmations sont peu nombreuses, deux essentiellement mais de très grande élévation spéculative, celle de la théologie trinitaire où trois sont un, et celle de la christologie où une personne est de deux natures, deux affirmations qui mettent en déroute les logiques seulement formelles :

la première réitère le monothéisme hérité d'Israël : un seul Dieu en son expression trinitaire ; la seconde est relative au seul médiateur et sauveur : Jésus Christ entre Dieu et les hommes. [...] Le christianisme [...] n'existe et ne se comprend que par [ces] deux affirmations conjointes qui le définissent. En dehors de ces deux affirmations, il n'y a point de religion chrétienne (214).

La raison philosophique ne se contente pas de la loi de non contradiction, ni d'oppositions entre des termes dont elle cherche plutôt les articulations. Breton s'est déjà occupé de la question dans son ouvrage de 1981, Unicité et monothéisme ${ }^{52}$. Trop bon lecteur des néoplatoniciens ${ }^{53}$, il sait déployer un discours dans lequel «un » et " autre » ne sont pas opposés. Les pages de L'avenir du Christianisme "Retour sur les notions d'unité et d'unicité » dans le chapitre 6 dédié à "Christianisme et espace interreligieux » importent à ce propos. Elles renvoient à la distinction sans contradiction entre foi et religion.

Les rapports entre la religion et « la foi en la croix » ne sont pas sans analogie avec les rapports des formes religieuses à l'élan mystique. De même que les rites et les représentations sensibilisent l'au-delà "mystique" qu'ils présupposent et qui les dépasse, de même la religion qui est et reste la nôtre a pour vocation de fournir à la foi et à la croix qui spécifie son élan le mode humain qu'elles requièrent pour exister parmi nous sur cette "terre oblique". En termes imagés : la foi doit toujours, par sa fonction critique, remonter les modalités religieuses qu'elle se donne ; la religion, quant à elle, se doit de faire descendre ou procéder jusqu'à nous, et selon notre humaine mesure, la foi et la croix qu'elle a mission de représenter (162).

L'avenir du Christianisme considère un certain nombre $\mathrm{d}^{\prime}$ » autres » du catholicisme, le monde protestant, le monde juif, le monde oriental, le bouddhisme par exemple. Le chapitre 8 du livre, le dernier, est intitulé précisément «Aujourd'hui : le christianisme et ses autres ». En vérité, cet aujourd'hui du christianisme n'est pas que d'aujourd'hui. En son cœur, il a toujours été une attitude de foi, d'attention à l'autre, le proche et l'autre absolu. En cela même, il s'accorde au don de l'Esprit qui rayonne en qui-

${ }^{52}$ BRETON, St. Unicité et monothéisme. Paris, Cerf, 1981

${ }^{53}$ Voir BRETON, St. Du principe : L'organisation contemporaine du pensable. Paris : Aubier-Montaigne, 1971. 
conque est à son écoute, même s'il n'en connaît pas le nom. Par contre, les déterminations théoriques ou dogmatiques des religions, outre à être des surcharges des origines, ne sont pas à même d'assurer par elles seules une communion spirituelle ou de foi. L'histoire enseigne qu'elles engendrent plus de certitudes et de violence que de vie et de charité, à moins que la foi ne les anime et leur donnent du large. L'avenir ou le futur du christianisme sera au sein des religions qui vivent selon l'Esprit, ce qui impliquera divers principes de communion (222-226) : un principe de charité qui fait tout le possible pour entendre de manière positive les dits de l'autre (un principe très ignacien, en réalité ${ }^{54}$ ), un principe d'intentionnalité qui reconnaît l'autre dans sa distance ou sa différence, un principe de joie et de synergie à travailler ensemble à partir des interrogations doctrinales « qui, pour le plus grand bien de chacun, permettent, à l'intérieur d'un même climat d'amitié, non seulement de s'informer de l'état des choses, mais, autant qu'il se peut, de lui accorder une place de choix dans sa réflexion » (225). Au cœur de cela, un chemin de conversion, celui de la Croix qui fait passer dans une vie nouvelle.

\section{Conclusion}

Comment parler de l'avenir du christianisme qui, ces derniers temps, a manifestement été mis à l'écart des sociétés occidentales ? Les années postconciliaires ont été importantes. Le livre La prise de parole de Michel de Certeau ${ }^{55}$ reflète ce qui y a été mis en cause. L'auteur y réfléchit sur les événements de mai 68. Le peuple français prend alors la parole, ou plutôt la récupère parce que le pouvoir politique s'en était approprié. N'en était-il pas de même dans l'Église ? Le travail fait par Vatican II en corrigeant un projet de décret sur l'Église le confirment. Les évêques avaient conscience de la situation. En Amérique Latine, en particulier en Argentine, se développa peu après une "théologie du peuple $»^{56}$. Le christianisme n'a-t-il pas été approprié par les théologiens qui discutent entre eux ? Où sont passés la simplicité et la radicalité humaine des paroles du Christ et de ses appels à l'action selon le cœur de Dieu ?

Cette perspective n'a-t-elle pas renouvelé cependant la vieille idée d'une division entre l'Évangile de Jésus dont le peuple est le destinataire et la théologie savante des intellectuels formés aux sciences de leur temps, entre

\footnotetext{
${ }^{54}$ Voir les Exercices spirituels de saint Ignace, n. 22.

${ }^{55}$ DE CERTEAU, M. La prise de parole : Pour une nouvelle culture. Paris : Desclée de Brouwer, 1968.

${ }^{56}$ Voir SCANNONE, J.C. La théologie du peuple : Les racines théologiques du pape François. Bruxelles : Lessius, 2017.
} 
l'Évangile qui appelle aujourd'hui et l'Église des théologiens qui discutent pour mettre à jour leurs cultures du passé ? Aux intellectuels, la logique des mots, la science, et au peuple, l'esprit, l'expérience évangélique ? Certes, le temps de la présence du Fils de l'homme au milieux de ses disciples n'est pas le temps de l'Église qui chemine dans le monde et qu'anime l'Esprit.

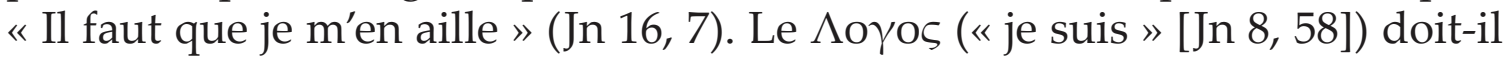
laisser la place à l'Esprit " qui souffle où il veut » $(\mathrm{Jn} 3,8)$ ? On connaît les aventures de cette conception de l'histoire. L'Esprit médiatise, il unit les différents sans les anéantir, il vit dans la reconnaissance d'autrui, de l'autre, dans l'accueil aux paroles simples. L'» à venir » du Christianisme ne nous est-il pas confié aujourd'hui, dans l'amour de l'autre reconnu pleinement dans sa dignité de frère?

Cela dit, le christianisme n'aura-t-il plus besoin de théologie ? Mais de quelle théologie ? La critique que nous avons entendue pointe en une direction claire : la théologie ne peut plus être d'une autorité qui en use sans pouvoir toucher le cœur du peuple de Dieu. Je ne me sens pas capable d'en dire plus. Le chantier est énorme. La seule certitude que j'ai est celle-ci : la théologie ne peut se déployer sans catégories, et l'élaboration de catégories est confiée au travail des philosophes. Bien sûr, beaucoup de chrétiens n'ont pas besoin de philosophie. Mais une théologie sans philosophie pourrait bien ne pas être digne de la Parole, ne pas respecter vraiment les personnes et ne pas pouvoir accompagner de manière saine ceux qui, en conscience, se décident de prendre leurs responsabilités dans la communauté ecclésiale.

\section{Bibliographie}

BELLET, M. Ceux qui perdent le foi. Paris: Desclée de Brouwer, 1965.

BELLET, M. Le Dieu pervers. Paris: Desclée de Brouwer, 1979.

BELLET, M. La quatrième hypothèse : Sur l'avenir du christianisme. Paris : Desclée de Brouwer, 2001.

BERGSON, H. Les deux sources de la morale et de la religion. Paris: Alcan, 1934.

BRETON, St. Du principe: L'organisation contemporaine du pensable. Paris: Aubier-Montaigne, 1971.

BRETON, St. Unicité et monothéisme. Paris, Cerf, 1981.

BRETON, St. L'avenir du christianisme. Paris: Desclée de Brouwer, 1999.

BRUAIRE, Cl. Philosophie du corps. Paris: Seuil, 1968.

CASTELLI, E. (éd.). Démythisation et morale. In: Archivio di filosofia, 1965. 
CASTELLI, E. (éd.). Démythisation et idéologie. In: Archivio di filosofia, 1973.

CHENU, M.-D. La théologie au douzième siècle. Paris: Vrin, 1957.

DE CERTEAU, M. La prise de parole : Pour une nouvelle culture. Paris: Desclée de Brouwer, 1968.

DE LUBAC, H. Exégèse médiévale: Les quatre sens de l'Écriture. Paris: Aubier, 1959-1964.

DE MAINDREVILLE, A. "François Roustang: Le troisième homme". In: Christus avril 2019, p. 114-115.

DECLOUX, S. "Vie religieuse et " Maîtres du soupçon »". In: Vies religieuses 50, 1978, p. 195-225.

Décrets de la trente-et-unième Congrégation générale de la Compagnie de Jésus. Prière et Vie, Toulouse, 1965.

DERRIDA, J. L'écriture et la différence. Paris: Seuil, 1967.

DERRIDA, J. Donner la mort, t. 1, La fausse monnaie. Paris, Galilée, 1991.

DERRIDA, J. "Foi et savoir". In: J. Derrida - G. VATTIMO, La religion, Paris : Seuil, 1996, p. 9-86.

FERRARIS, M. Dove sei ? Ontologia del telefonino. Milano: Bompiani, 2005.

FERRY, L. L'homme-Dieu, ou le sens de la vie. Paris: Grasset, 1996.

FOUCAULT, M. Les mots et les choses. Paris: Gallimard, 1966.

FOUILLOUX, Ét. “L'affaire du « Troisième homme »". In: E.-A. Roustang et al. (éd.), François Roustang.

GAUCHET, M. Le désenchantement du monde: Une histoire politique de la religion. Paris: Gallimard, 1985.

GILSON, Ét. Le thomisme: Introduction à la philosophie de saint Thomas d'Aquin. Paris: Vrin, $1944^{5}$.

GODIN, H. - DANIEL, Y. France, pays de mission. Paris: Cerf, 1943.

HEIDEGGER, M. Introduction à la métaphysique. Paris: Gallimard, 1967.

HEIDEGGER, M. Être et temps. trad. hors commerce de Martineau. Paris: Authentica, 1985.

HENRY, M. Philosophie et phénoménologie du corps. Paris: PUF, 1965.

HUSSERL, Ed. La crise de l'humanité européenne et la philosophie. Paris: Hatier, 1992.

HUSSERL, Ed. Recherches logiques, t. 2, Recherches sur la phénoménologie et la théorie de la connaissance. Première partie. Recherches I et II. Paris, PUF, $1996^{2}$.

IGNACE DE LOYOLA, Exercices spirituels.

JANICAUD, D. Le tournant théologique de la phénoménologie française. Combas: L'Éclat, 1991.

KIERKEGAARD, S. "Sur une tombe". In : ID., CEuvres complètes, t. VIII, Paris : L'Orante, 1979, p. 61-89. 
LANGLOIS, Cl. “Un long compagnonnage. François Roustang et Michel de Certeau à Christus". In: E.-A. Roustang et al. (éd.), François Roustang, p. 63-98.

LÉVINAS, Em. Totalité et infini: Essai sur l'extériorité. Den Haag: Martin Nijhoff, 1990. MARION, J.-L. Étant donné : Essai d'une phénoménologie de la donation. Paris: PUF, 1997.

MERLEAU-PONTY, M. Le visible et l'invisible. Paris: Gallimard, 1964.

MOINGT, J., L'esprit du christianisme. Paris: Temps Présent, 2018.

MOREL, G. Le sens de l'existence selon saint Jean de la Croix. 3 vol. Paris: Aubier, 1960-1961.

MOREL, G. Problèmes actuels de religion. Paris: Aubier-Montaigne, 1968.

MOREL, G. Nietzsche : Introduction à une première lecture. 3 vol. Paris: Aubier-Montaigne, 1970-1971.

MOREL, G. Questions d'homme. 3 vol., Paris : Aubier-Montaigne, 1976-1978.

PLATON, Timée.

RICCEUR, P. L'homme faillible. Paris: Aubier-Montaigne, 1960.

RICEEUR, P. De l'interprétation: Essai sur Freud. Paris: Seuil, 1965.

RICEEUR, P. Le conflit des interprétations: Essais d'herméneutique. Paris: Seuil, 1969.

ROUSTANG, E.-A. et al. (éd.). François Roustang: Le troisième homme, entre rupture personnelle et crise catholique. Paris: Odile Jacob, 2019.

ROUSTANG, Fr. “Le troisième homme”. In: Christus 13, 1966, p. 561-567.

SCANNONE, J. C. La théologie du peuple : Les racines théologiques du pape François. Bruxelles: Lessius, 2017.

THOMAS D'AQUIN, Somme de théologie, Ia-IIae, Prologue.

WEBER, M. L'éthique protestante et l'esprit du capitalisme. Paris: Gallimard, 2003.

WEIL, S. Attente de Dieu. Paris: Fayard, 1966.

Endereço do Autor:

Via del Seminario, 120

00186 Roma - Itália

gilbert@unigre.it 